\title{
Tratamiento de mordida cruzada anterior con aparato de tracción extraoral Máscara Delaire en paciente con estrabismo: Reporte de caso
}

\section{Anterior crossbite treatment with headgear: Delaire mask, in a patient with strabismus: Case report}

\author{
Julieth Alejandra Ariza ${ }^{1}$ (D) $\mid$ Angela Patricia Flórez ${ }^{1}$ (D) $\mid$ Martha Elena Hurtado ${ }^{1}$ (D)
}

\section{G ACCESO ABIERTO}

${ }^{1}$ Universidad Cooperativa de Colombia, Facultad de Salud, Departamento de Ortodoncia, Bogotá, Colombia.

\section{Citación}

Ariza JA, Flórez AP, Hurtado ME. Tratamiento de mordida cruzada anterior con aparato de tracción extraoral Máscara Delaire en paciente con estrabismo: Reporte de caso. Rev Estomatol. 2019;27(2):18-24. DOI: $10.25100 /$ re.v28i.1.8704

\section{Correspondência}

Martha Elena Hurtado, Dirección: Calle 169 B \# 75-73, Bogotá, Colombia. Celular: 3115539212. Email:

martha.hurtado@campuscc.edu.co

\section{Copyright:}

(C) Universidad del Valle.

\begin{abstract}
RESUMEN
En la maloclusión clase III con mordida cruzada anterior existe una alteración en la relación máxilo-mandibular: retrognatismo maxilar, prognatismo mandibular o combinada, esta desarmonía puede alterar la estética facial. Si se interviene a una edad temprana se puede lograr la normalidad de la funciones y armonía en la relación de los maxilares, llevando a mejorar la estética del paciente. Es importante tener en cuenta el factor etiológico para establecer un adecuado plan de tratamiento y lograr estabilidad a largo plazo. La etiología es multifactorial; hereditaria y ambiental (hábitos, agrandamiento de las amígdalas y postura mandibular). Existen varias opciones de tratamiento dependiendo de la edad como ortopedia maxilar, ortodoncia y cirugía ortognática. Este reporte de caso, presenta una paciente de 9 años y 7 meses de edad, género femenino, con estrabismo, cuya madre consulta porque "se le está saliendo la mandíbula"; los hallazgos clínicos y cefalométricos determinan una maloclusión clase III con retrognatismo maxilar, mordida cruzada anterior, crecimiento vertical, respiración mixta con predominio nasal, deglución atípica y sigmatismo. El tratamiento que se realizó en este paciente fue en 2 fases: en la primera ortopedia mecánica con Hyrax y máscara facial de Delaire por 11 meses y en la segunda fase aparato de ortopedia funcional SN3 (pequeñas aletas inferiores). Los cambios obtenidos con máscara facial de Delaire se evidencian a nivel facial, dental y esquelético, sin comprometer la alteración de estrabismo que la paciente presenta y maneja con gafas.
\end{abstract}

PALABRAS CLAVE

Maloclusión de Angle Clase III; estrabismo; desarrollo maxilofacial; ortodoncia interceptiva; aparatos de tracción extraoral..

\section{ABSTRACT}

In Class III malocclusion with anterior crossbite exist an altered relationship between maxilla and mandible: maxillary retrognathism, mandibular prognathism or a combination of these; this disharmony can alter facial aesthetics. The etiology is multifactorial; hereditary and environmental (habits, enlarged tonsils and mandibular posture). Considering the etiological factor is crucial to establish an adequate treatment plan and achieve long-term stability. An intervention at an early age can achieve a normal function and harmony in the relationship of the jaws, thus improving the aesthetics of the patient. There are several treatment options depending on age such as maxillary orthopedics, orthodontics and orthognathic surgery. This case report depicts a 9-year-old and 7-month-old female patient, with strabismus, whose mother consults because "her jaw is getting out"; the clinical and cephalometric findings determine a class III malocclusion with maxillary retrognathism, anterior cross bite, vertical growth, mixed breathing with nasal predominance, atypical swallowing and sigmatism. The treatment was developed in 2 phases: first mechanical orthopedics with Hyrax and Delaire's facial mask for 11 months, in the second phase SN3 functional orthopedic appliance (small lower fins). The changes obtained with Delaire's facial mask are evidenced at the facial, dental and skeletal level, without compromising the strabismus alteration that the patient presents and handles with glasses.

\section{KEYWORDS}

Malocclusion; Angle Class III; strabismus; maxillofacial development; ortodontic interceptive; extraoral traction appliances. 


\section{INTRODUCCIÓN}

La maloclusión clase III con mordida cruzada anterior es una alteración difícil de corregir, afecta las funciones orales y la estética del paciente; para elegir un tratamiento adecuado es importante tener en cuenta la etiología, realizar un diagnóstico esquelético, dental y funcional acertado, con el objetivo de lograr resultados óptimos y estables.

La prevalencia de la maloclusión clase III según el estudio epidemiológico ENSAB IV 2013-2014, realizado en población colombiana, demuestra que los niños en edades de 5 años presentan escalón mesial derecho en un $74.89 \%$, del lado izquierdo $72.59 \%$, observando resultados similares en niñas que presentan escalón mesial derecho en un $70.52 \%$ y del lado izquierdo $68.61 \%$, evidenciando la alta incidencia del escalón mesial que puede llevar en algunos casos a una relación molar clase III en dentición permanente. ${ }^{1}$

En un estudio realizado en la Universidad Cooperativa de Colombia sobre la prevalencia de la maloclusión en tres planos del espacio en pacientes diagnosticados con defectos del habla en las clínicas de la especialización de ortopedia funcional y ortodoncia, la mayor prevalencia fue la clase I esquelética (34.1\%) seguida de la maloclusión clase III $(31.7 \%)$ y en menor frecuencia la clase II $(7.3 \%){ }^{2}$

La maloclusión clase III es una alteración en la posición sagital de los maxilares: por retrognatismo maxilar, prognatismo mandibular o una combinación de ambos. La causa de la maloclusión puede ser dentoalveolar, esquelética, o ambas. Los pacientes clase III se caracterizan por presentar alteraciones faciales, esqueléticas, funcionales $\mathrm{y}$ dentales (perfil facial cóncavo o recto con tendencia a cóncavo, tercio medio facial deprimido, proquelia inferior, deglución atípica, posición lingual baja, mordida cruzada anterior y/o posterior). ${ }^{3}$

La etiología de la maloclusión clase III es multifactorial: hereditaria con gran influencia ambiental (hábitos, agrandamiento de las amígdalas y postura mandibular), congénita y adquirida, la última puede deberse a síndromes como Marfan, androgenial, acromegalia, labio y paladar hendido, síndrome de Down. ${ }^{3,4}$
La clase III por retrognatismo maxilar con hipoplasia maxilar es la falta de crecimiento en sentido sagital, vertical y transversal del maxilar superior, compromete el tercio medio y la estética facial al presentar depresión de los pómulos; cuando no se trata a edad temprana puede generar efectos psicológicos nocivos para el paciente, por las alteraciones físicas y funcionales que se desarrollan por esta deficiencia. ${ }^{5}$

Durante el tratamiento se debe tener en cuenta que el crecimiento del maxilar en la sutura media palatina se da hasta los 7 años y la formación del tejido conectivo tiene un periodo más prolongado, por esta razón entre más temprano se realice el tratamiento mayor es el crecimiento del maxilar en profundidad y mayor va a ser la respuesta del hueso ante las fuerzas de ortopedia. ${ }^{3}$ Este resultado se debe a que el tipo de crecimiento del maxilar superior es intramembranoso con proliferación de tejido conectivo sutural, reabsorción, translación y crecimiento en $\mathrm{V}$; el crecimiento en sentido anteroposterior del maxilar finaliza en la etapa de la pubertad, mientras que el vertical continúa. La literatura refiere que el pico del crecimiento del maxilar para niñas es entre los 10 y 12 años, mientras que para los niños es 1a 3 años más tarde. 5

La maloclusión clase III puede ser tratadada de diversas maneras, el tratamiento depende del origen de la maloclusión, del patrón de crecimiento, de la edad esquelética, de la motivación del paciente, del diagnóstico y de la experiencia clínica del especialista que lo va a realizar, entre otros; el tratamiento busca estimular el crecimiento maxilar, controlar y/o redirigir el crecimiento mandibular. A una edad temprana es posible tratar con ortopedia funcional para lo cual se pueden emplear aparatos como el Regulador de función Frankel III, el Bionator de Balters inversor, el activador de Klammt para clase III; así como ortopedia mecánica con máscara facial de protracción combinada con aparatos intraorales expansores tipo Hyrax con bandas o con férula oclusal en acrílico. ${ }^{6}$

La primera máscara facial fue creada por Oppenheim en 1944, posteriormente fue modificada por Delaire y Pettit haciéndola más confortable (5). La máscara facial de Delaire creada por el profesor Delaire en 1968 en Francia, tiene como objetivo la corrección de la clase III mediante la protracción 
maxilar en pacientes en edad temprana. El éxito del tratamiento consiste en la orientación del maxilar en relación con la implantación craneal superior permitiendo mejorar el crecimiento en la parte anterior premaxilar y proceso frontomaxilar. En cuanto a la mandíbula modifica la dirección y controla el crecimiento del cartílago condilar, logrando un equilibrio maxilo-mandibular. ${ }^{7}$

La máscara facial es un aparato mecánico que tiene buenos resultados en la clase III de leve a moderada con retrusión del maxilar; la edad más adecuada para iniciar el tratamiento es antes de los nueve años en dentición mixta temprana, para lograr más cambios esqueléticos y menos movimientos de los dientes; a menor edad se obtienen mejores resultados. ${ }^{8}$

Se requieren 3 fases de tratamiento: Expansión, Protracción y Retención. Durante la expansión se afecta tanto la sutura media palatina como las suturas circunmaxilares. Los aparatos que se utilizan en la expansión deben ser fijos tipo Hyrax con bandas o con férula oclusal acrílica, indicados en oclusión borde a borde o mordidas cruzadas. ${ }^{8}$

Después de estimuladas las suturas circunmaxilares se realiza la protracción con la máscara facial, se emplean fuerzas de 400 a $500 \mathrm{~g}$ estimulando el movimiento anterior del maxilar, para obtener cambios sagitales del complejo cráneo facial; esta fase dura aproximadamente de 8-12 meses. Los efectos de esta mecánica son: el desplazamiento anterior del maxilar de 2 a $4 \mathrm{~mm}$, rotación horaria de la mandíbula, proinclinación de incisivos superiores y retroinclinación de incisivos inferiores. $4,8,9$

Por último, la fase de retención que es muy importante para mantener los objetivos conseguidos, cuando se corrija el resalte y la sobremordida, se debe usar la máscara día de por medio, preferiblemente en la noche por un periodo de 4-6 meses y posteriormente utilizar un aparato funcional como el Regulador funcional de Frankel.

\section{PRESENTACIÓN DEL CASO}

Paciente femenino de 9 años y 7 meses de edad, se presenta a la clínica de ortopedia de la Universidad Cooperativa de Colombia sede Bogotá, en el 2018 en compañía de la mamá; a nivel general es una paciente sana sin compromiso sistémico, el motivo de consulta referido por la madre "se le está saliendo la mandíbula y se le perdió el aparato que tenía antes".

La madre relata antecedente de tratamiento con ortopedia "placa de Hawley con tornillo", en el que no hubo colaboración de la paciente y de los padres para su total adherencia al tratamiento, tuvo una duración de 1 año aproximadamente.

$\mathrm{Al}$ examen facial la paciente presenta un tipo facial mesoprosopo, perfil recto, depresión del tercio medio de la cara, proquelia superior e inferior, estrabismo (Figura 1).

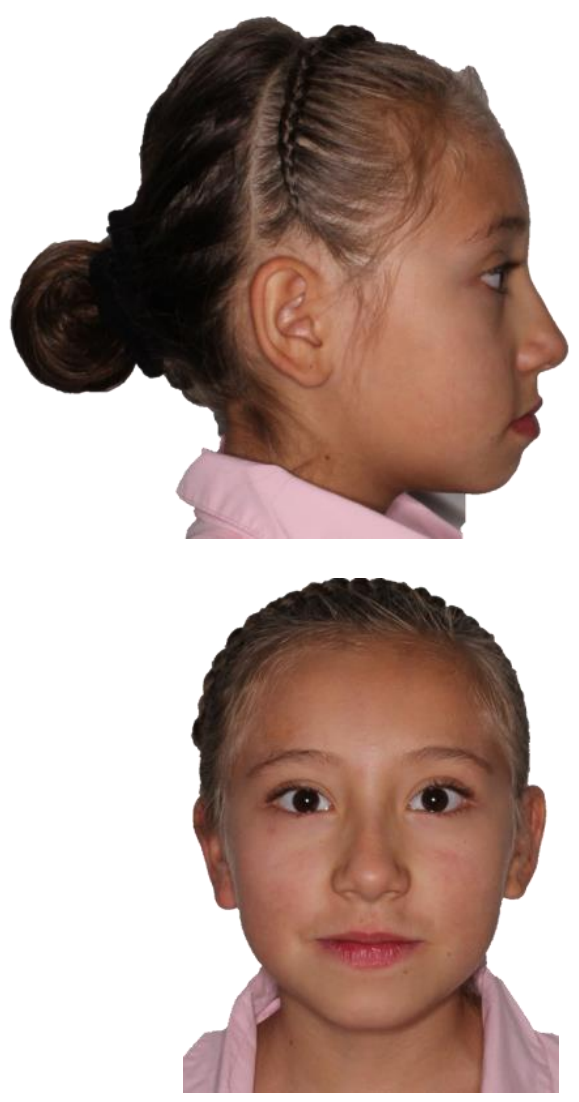

Figure 1. Vista frontal y lateral iniciales de la paciente.

A nivel intraoral presenta dentición mixta tardía, con relación canina y molar bilateral clase III, mordida cruzada anterior $(-2 \mathrm{~mm})$, overbite de $35 \%$, micrognatismo leve superior e inferior, apiñamiento leve superior, mordida cruzada posterior derecha (Figura 2). 



Figure 2. Fotografías intraorales iniciales.

Al examen funcional presenta deglución atípica y patrón respiratorio mixto con predominio nasal. Al análisis esquelético presenta clase III esquelética con retrognatismo maxilar leve, ortognatismo mandibular, base de cráneo corta y crecimiento vertical. Proinclinación de incisivos superiores e inferiores (Figura 3). Las medidas cefalométricas iniciales se observan en la Tabla 1.



Figure 3. Radiografía lateral de cráneo inicial.
Tabla 1. Medidas cefalométricas iniciales.

\begin{tabular}{lcc}
\hline \multicolumn{1}{c}{$\begin{array}{c}\text { Datos } \\
\text { cefalométricos }\end{array}$} & Norma & Paciente \\
\hline SNA & $80-84^{\circ}$ & $79^{\circ}$ \\
SNB & $78-82^{\circ}$ & $80^{\circ}$ \\
\hline ANB & $2^{\circ}$ & $0^{\circ}$ \\
\hline SN-PM & $30-34^{\circ}$ & $44^{\circ}$ \\
\hline Lineal NS & $68-74 \mathrm{~mm}^{\circ}$ & $55 \mathrm{~mm}$ \\
\hline Angulo Is-SN & $100-104^{\circ}$ & $107^{\circ}$ \\
\hline Angulo Ii -PM & $87-93^{\circ}$ & $90^{\circ}$ \\
\hline Is-Ii (Angulo & $124-136^{\circ}$ & $119^{\circ}$ \\
Interincisivo) & & \\
\hline
\end{tabular}

Los objetivos del tratamiento elegidos fueron: Vigilar el perfil facial, corregir la depresión del tercio medio de la cara; corregir las relaciones canina y molar clase III, la mordida cruzada anterior, el overbite; lograr avance maxilar y la armonía maxilo-mandibular, así como controlar crecimiento vertical.

El tratamiento se realizó en 2 fases: En la primera fase se colocó un aparato fijo tipo Hyrax , con topes en resina en los dientes 16 y 26 para realizar el levantamiento de la mordida y facilitar la protracción, acompañado por máscara facial de Delaire (Figura 4), escogida porque este tipo de máscara no contaba con vástago central vertical que pudiera comprometer el estrabismo que presentaba la paciente.

El protocolo que se implementó para soltar la sutura media palatina y las suturas circunmaxilares fue de expansión y contracción del tornillo Hyrax, durante un mes, de manera intercalada, iniciando en la primera semana con la expansión de un cuarto de vuelta diario; continuando en la segunda semana con la contracción, cerrando un cuarto de vuelta diario; seguido de otra semana de expansión y finalizando con la semana de contracción. 

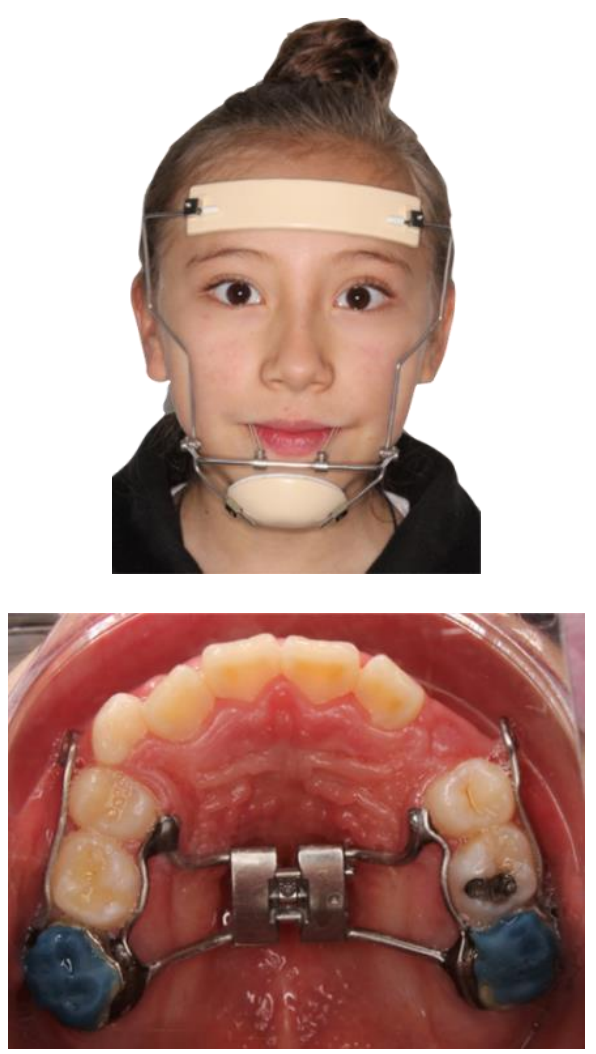

Figure 4. Aparatología tipo Hyrax y Máscara Facial.

Posteriormente se inició la protracción del maxilar superior con elásticos que conectaban los ganchos de protracción del aparato intraoral a la máscara, calibre 5/16 pesados que generaron una fuerza de $300 \mathrm{gr}$; la fuerza se fue incrementando de acuerdo con las condiciones oclusales en que llegaba la paciente a cada control mensual. A los 11 meses de tratamiento se observan cambios clínicos faciales y dentales significativos, por lo que se decide solicitar ayudas diagnósticas de progreso.

En los nuevos análisis clínicos y cefalométricos se observa corrección de la depresión del tercio medio de la cara, perfil más armónico; relación canina y molar clase I bilateral, corrección de la mordida cruzada anterior con un overjet de $2 \mathrm{~mm}$ y overbite de $30 \%$, proinclinación de incisivos superiores y retroinclinación de incisivos inferiores; avance del maxilar superior, con corrección de la clase III esquelética, control del patrón de crecimiento vertical (Figuras 5, 6, 7). Los cambios de las medidas cefalométricas finales se observan en la Tabla 2 .

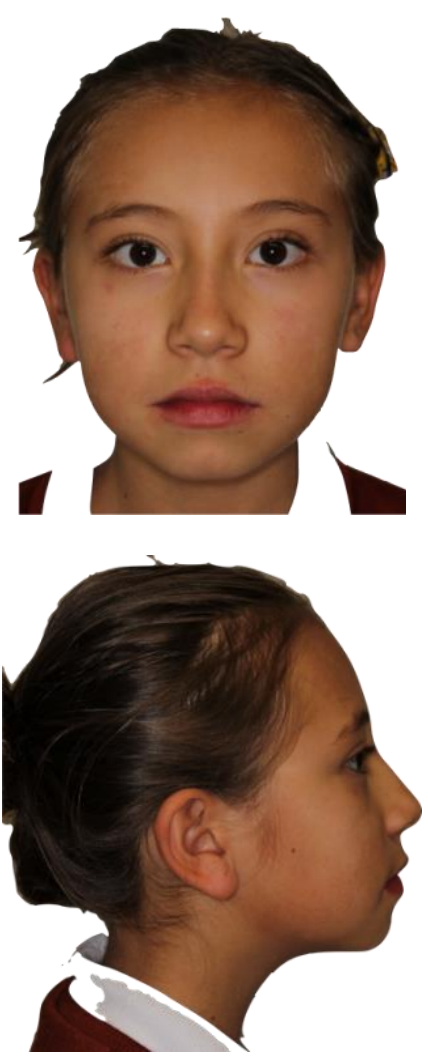

Figure 5. Vista frontal y lateral finales de la paciente.

Tabla 2. Medidas cefalométricas finales.

\begin{tabular}{lcc}
\hline \multicolumn{1}{c}{$\begin{array}{c}\text { Datos } \\
\text { cefalométricos }\end{array}$} & Norma & Paciente \\
\hline SNA & $80-84^{\circ}$ & $82.5^{\circ}$ \\
SNB & $78-82^{\circ}$ & $80^{\circ}$ \\
\hline ANB & $2^{\circ}$ & $2.5^{\circ}$ \\
SN-PM & $30-34^{\circ}$ & $44^{\circ}$ \\
\hline Lineal NS & $68-74 \mathrm{~mm}^{\circ}$ & $56 \mathrm{~mm}^{\circ}$ \\
\hline Angulo Is-SN & $100-104^{\circ}$ & $108^{\circ}$ \\
\hline Angulo Ii -PM & $87-93^{\circ}$ & $83^{\circ}$ \\
\hline $\begin{array}{l}\text { Is-Ii (Angulo } \\
\text { Interincisivo) }\end{array}$ & $124-136^{\circ}$ & $126^{\circ}$ \\
\hline
\end{tabular}



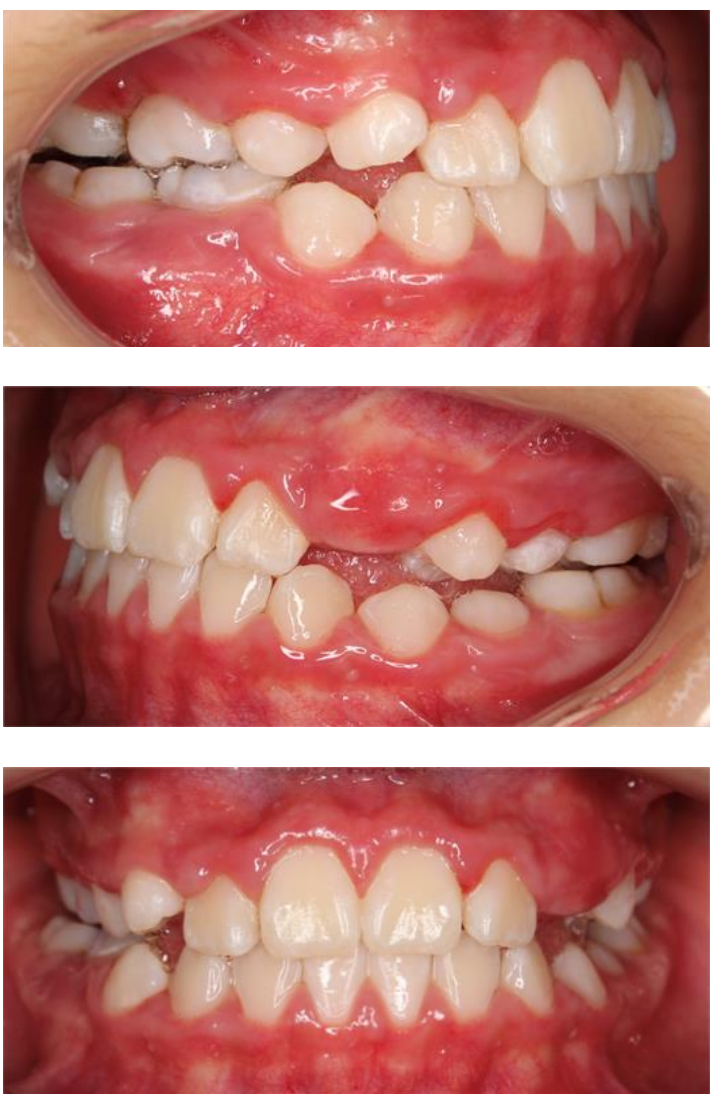

Figure 6. Fotografías intraorales finales.



Figura 7. Radiografía lateral de cráneo final.

Es importante destacar la acción que tuvo la máscara en corto tiempo al lograr los objetivos propuestos, teniendo en cuenta que, si este tipo de alteraciones esqueléticas no se corrigen a edad temprana, pueden generar problemas psicosociales debido a las características faciales, que a futuro solo se pueden resolver con cirugía ortognática y no todos los pacientes tienen el acceso a este tipo de procedimientos.

En la fase 2 la paciente continúa el tratamiento con aparatología ortopédica funcional para mantener los cambios; el aparato elegido fue el SN3 (pequeñas aletas inferiores), ya que con este aparato se controla el crecimiento vertical, con la barra ondulada se maneja la posición de la lengua para corregir la función deglutoria y permite seguir acompañando el crecimiento transversal de los $\operatorname{arcos}$ (Figura 8).
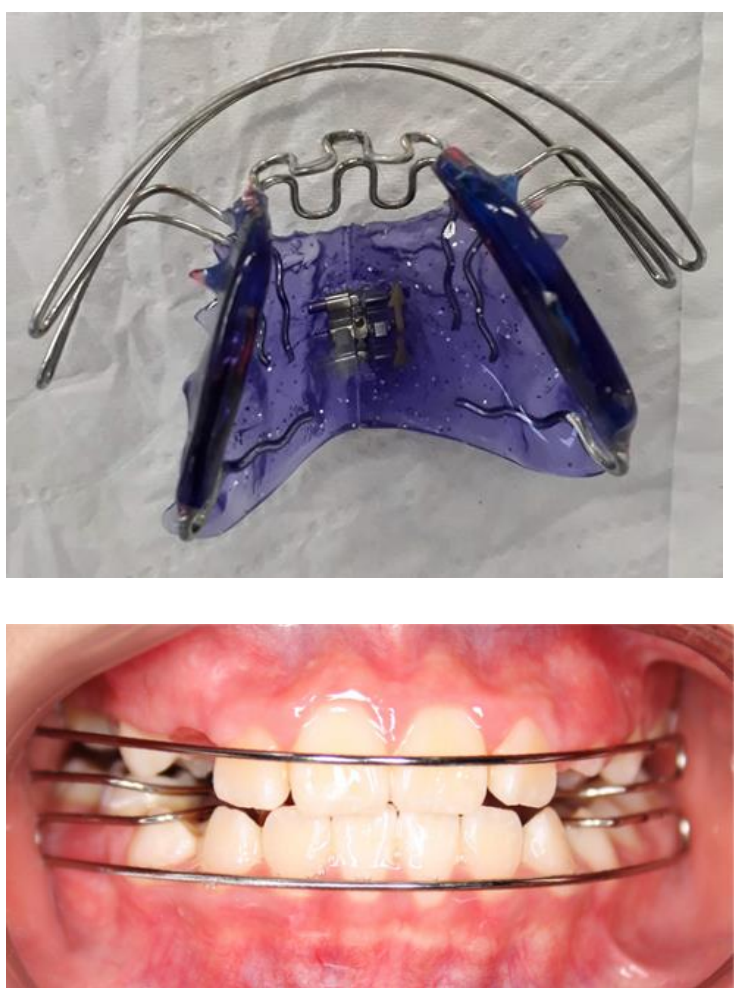

Figura 8. Simoes Network 3 (SN3). Aparatología de retención.

\section{DISCUSIÓN}

En el tratamiento con Hyrax y máscara facial de Delaire se logró un avance maxilar de $2 \mathrm{~mm}$ en un periodo de tiempo de 11 meses, donde su eficacia se sustenta en la literatura científica con investigaciones como las de Ngan $\mathrm{P}$ y cols, Martinez R y cols, Cha B y cols, quienes plantean que con la máscara facial se da una protracción de 2-4 mm durante 8 a 12 meses de tratamiento. ${ }^{4,8,9}$

La paciente del presente reporte de caso tiene una 
edad de 9 años, respondió de manera satisfactoria al tratamiento, observando cambios faciales, dentales y esqueléticos; Martinez R y Cols sugieren que la edad ideal para tratar esta maloclusión con ortopedia mecánica es en menores de 9 años para lograr cambios esqueléticos, evitar mayores compensaciones dentales y compromiso facial que puede alterar psicológicamente los pacientes. ${ }^{8}$

Es importante tener en cuenta que uno de los efectos de la máscara facial es la proinclinación de incisivos susperiores y retroinclinación de incisivos inferiores lo que se evidenció en el presente reporte de caso. ${ }^{5,9}$

Es fundamental que al intervenir esta maloclusión con ortopédia mecánica se realice la fase de retención con aparatos de ortopedia funcional, por esta razón en la segunda fase se colocó el SN3 para mantener los resultados obtenidos y manejar la parte funcional, como lo indica Martinez R y Cols en su reporte de caso. ${ }^{8}$

\section{CONCLUSIÓN}

La máscara facial es una excelente alternativa para corregir la maloclusión clase III por retrognatismo maxilar y depresión del tercio medio facial, especialmente en edades tempranas donde las suturas circunmaxilares aún son susceptibles de separación y es posible la corrección esquelética. Los cambios obtenidos con máscara facial se evidencian a nivel facial, dental y esquelético.

\section{CONFLICTO DE INTERÉS}

Las autoras declaran que no existe conflicto de intereses para la publicación del manuscrito, ni se persiguen intereses económicos por el mismo.

\section{FUENTE DE FINANCIAMIENTO}

Este proyecto no tuvo financiación.

\section{REFERENCIAS}

1. Ministerio de Salud y protección social. IV Estudio Nacional de Salud Bucal ENSAB IV. Ministerio de salud y protección social Colombia. 2013-2014

2. Peña M, Rojas M del P, Tirado A, Benavides B, Hurtado M, Ruíz A. Prevalencia de la maloclusión en tres planos del espacio en pacientes diagnosticados con defectos del habla en las clínicas de la especialización de ortopedia funcional y ortodoncia de la Universidad Cooperativa de Colombia. Rev. Estomatol. 2014; 22(1):26-32.

3. Espinar E, Ruiz M, Ortega H, Llamas J, Barrera J, Solano J. Tratamiento temprano de las Clases III. Revista Esp Ortodoncia. 2011;41:79-89

4. Ngan P, Moon W. Evolution of class III treatment in orthodontics. Am J Orthod Dentofacial Orthop 2015;148:22-36

5. Meneses D, Botero P. Alternativas para el tratamiento de hipoplasias maxilares. Rev CES Odont. 2012;25(1)64-81.

6. Gencer D, Kaygisiz E, Yüksel S, Tortop T. Comparison of doublé-plate appliance/facemask combination and facemask therapy in treating Class III malocclusions. Angle Orthod. 2015;85:278-283.

7. Puig L, Altunaga A, Hidalgo A. La máscara facial ortopédica: su importancia en el tratamiento de la clase III de angle. Rev Arch Med de Camagüey. 2002;6(4).

8. Martinez R, Aristizabal J. Maloclusión Clase III: Diagnóstico y Tratamiento Ortopédico. Revisión de Literatura y Reporte de Caso. Rev CES 2016. Vol.3, N²

9. Cha B, Choi D,Ngan P,Jost P, Kim S, Jang I. Maxillary protraction with miniplates providing skeletal anchorage in a growing Class III patient. Am J Orthod Dentofacial Orthop 2011;139:99-112

10. Soldevilla L, Aliaga A. Tratamiento de una maloclusión clase III en dentición decidua y mixta con expansión rápida rápida palatina y mascara facial. Odontol. Sanmarquina 2011; 14(1): 26-29

11. Tortop T,Kaygisızb E, Gencerc D, Yuksela S, Atalay Z. Modified tandem traction bow appliance compared with facemask therapy in treating Class III malocclusions. Angle Orthod. 2014;84:642-648.

12. Armas M, Schaffry M, Ubilla W, Moreira T. Indicaciones de la aparatología ortodòntica extra oral en pacientes en etapa de crecimiento de 6 a 12 años de edad. Rev. Cient. Univ. Odontol. Dominic. 2018. 6 (1): 69-83.

13. Cobourne M. Early treatment for class III malocclusion. J Orthod. 2016 (43): 159-160.

14. Stocker B, Willmann J, Wilmes B, Vasudavan S, Drescher D. Wear-time recording during early Class III facemask treatment using TheraMon chip technology. Am J Orthod Dentofacial Orthop 2016;150:533-40.

15. Willmann J, Nienkemper M, Tarraf N, Wilmes B, Drescher D. Early Class III treatment with Hybrid-Hyrax -Facemask in comparison to Hybrid-HyraxMentoplate - skeletal and dental outcomes. Willmann et al. Progress in Orthodontics. 2018; 19-42.

16. Acosta I. Tratamiento de las maloclusiones clase III con el uso de mascara facial. Revisión bibliográfica. Universidad de Carabobo 2014 